\title{
SOME CORE LEGAL CHALLENGES OF DIGITAL TRANSFORMATION
}

\author{
Reinhard Riedl
}

Abstract: The digital transformation creates many trends that will eventually reshape large parts of the economy and the society. We discuss the core trends and their consequences. Thereby, we address both industry and government. For each trend, we explain some of the most immediate challenges for legislation. In some areas adaptions of regulations will suffice, while in other areas we need fundamentally new concepts and even paradigm changes.

\section{Table of contents}
A. The Nature of Digital Transformation 273
B. Digital Metatrends
C. ten firing Stages of the digital transformation rocket. 281
D. Trends in Government 287
E. The dangers of digital transformation 289
F. The true challenges. 291

\section{A. THE NATURE OF DIGITAL TRANSFORMATION}

The digital transformation is not a buzzword, but a complex process taking place in many places and aspects. Several of the metaphors used to describe the process are misleading: For example, the digital transformation has a short-term disruptive effect only in exceptional cases. As another example, the acceleration of change plays a subordinate role in concrete contexts. Nevertheless, digitization is not «business as usual». If applied properly, it fundamentally changes the scope of action. Therefore, it makes sense to use the term «digital transformation» that indicates that we are not talking of 
an introduction / replacement of technologies but rather about a transformation that exploits the new opportunities created by the new information and communication technologies (ICT).

In the long run as well as in the global scale, this transformation indeed will have very «disruptive» effects. We may infer this from what we have learned in the past, and we may deduce it from the obvious observation, that it has the potential to change the way we work and live. In fact, detestation increases the freedom to perform tasks significantly thus enabling a diverse set of benefits if we exploit this new freedom. Furthermore, from the point of view of society, the digitation indeed accelerates change because it occurs and interacts in extremely high parallelism.

At its core, digital transformation is about the introduction of digital tools that support or completely take over human work. This turns it into an enabler for all actors in society - an enabler for further development of individuals, of organizations, of disciplines, and of government. Of course, the list of enabled entities includes criminals, revolutionaries and terrorists. And like many enablers, the digital transformation creates both chances and risks. Consequently, it may be beneficial or detrimental for the enabled entities depending on how they and others use the tools provided. In fact, it is not only the use of tools, but also - and in some cases first and foremost - the design of tools that will have a strong impact on the effects. There is some evidence for an interplay between technological design and the application of technology, which is strengthened in all cases where there is a strong affinity between design principles of technology and of the application domain, such as between principle to distribute intelligence at the periphery (as a tradition in real time systems) and the principle of subsidiarity (as an old organizational principle in government). Thus, it is technology and its usage - or more precisely: technology, its appropriation, and its usage - that influence each other and jointly drive the digital transformation.

While local short time effects may be small, there is little doubt about the overrange reach of the midterm to long-term change induced by the digital transformation. It will particularly concern social coexistence and it will challenge all actors, because on the one hand the appropriation of tools is not always easy and on the other hand one can hardly escape the digitalized world with the lots of other digitally competent people. 
The latter means that we are not free to say no to the use of the new digital tools and services.

As an example, let us look at the domain of healthcare. Its digital transformation enables patients to assume more responsibility for their own health. Health professionals are empowered to do better work for the benefit of patients. The operations of hospitals and ambulances can become both more efficient and improve the quality and patient value of services. Those, who finance the services, can reduce the administrative costs and make improved cost-benefit calculations. And decision makers in politics may invest taxpayers' money more effectively. Furthermore, even if we decide to neglect these possibilities, we shall observe that specialists on ICT and the usage of ICT will enter the healthcare sector by way of transversal innovations. The latter will lead to creative destruction of existing structures.

The depicted empowerment will change the labor market in healthcare. Some job profiles will disappear, more job profiles will be changed significantly, and new job profiles will be created - for example, the job of health data coaches for patients and for professionals. New skills and abilities will be required from everyone in healthcare - that is from patients and healthcare professionals alike, as well as from employees in companies and government agencies related to healthcare. Along with these new skills new human judgement capabilities will be required and new legal and ethical questions will arise, because laws and ethical rules of thumb cannot go unchanged if skills, activities and options change dramatically.

It follows from the above that new risks and new governance challenges will be created. For example, legislation will have to deal with questions arising from dramatically improved transparency about individual patients and to individual professionals. Among many other forms of impact this may call into question the principle of solidarity. And it will for sure call on policymakers to consciously safeguard patients. Equally important, it will force us to formulate fundamental principles on the autonomy of professionals in healthcare as we will soon be able to monitor nearly all their activities and to evaluate these activities with the support of machine learning. Furthermore, the relationship between service financiers, service organizers and service providers in healthcare will be changed. One issue particularly highlights the impact of the emerging new transparency for the interplay of actors: How shall we deal with deviations from 
standard treatments without justification from previous experience? This question will hardly allow for generic answers as the interplay of standardization and individualization is driving the progress of medicine. This interplay is well understood in high end medicine, but much more unclear in the field of general practitioners lacks.

The above considerations demonstrate that everyone is challenged in the domain of healthcare. Patients are made aware of the effects of their own behavior and can now compare themselves more adequately with others. At the same time, they are confronted with the fact that the protection of their privacy requires much more care and is only possible to a limited extent. Professionals are challenged because automation moves activities to less educated job profiles and better educated profiles are confronted with more challenging tasks, both with respect to cognitive and with respect to emotional intelligence. And decision makers on all levels - including government - are challenged by the emerging new possibilities for information-oriented decision making.

Most of these challenges appear both in normal life and life in threatening crises. The digital transformation of just one single domain such as healthcare concerns nearly all areas of politics. For example, it is known for long that social status has a big impact on health. Currently, digitally enabled research is increasing our knowledge about specific aspects and cause-and-effect relationships. In the future, there will be new possibilities for targeted interventions to decrease the negative impact of low social status Thus, the transformation of healthcare provides new instruments for effective social politics. Similar holds for education, for the labor market, and for other domains of politics.

Summing up, locally and within short time, the changes caused by digitation are small, manageable and even easily overlooked. Globally and in the long run however, the basis for action will change radically. The expected effect is so great because digital change is taking place simultaneously in so many areas whereby it radically transforms practice and leads to totally new capabilities. It is not only more complex, but also much more comprehensive than earlier technologically induced changes. These, too, had a versatile effect, such as the invention of the steam engine, but never as comprehensive on society as the digital transformation.

Despite of the evidence about its broad impact, the transformation process is by no means deterministic. It is characterized by hardly predictable path dependencies. For 
example, it depends on cultural developments with respect to lifestyles and social values. Today we cannot predict where the journey will take us, and yet we must ensure, though we benefit as a society from these changes and that we can mitigate risks. These risks include the risk of wrong regulations, that either block progress or on the contrary open the doors for misuse - or doing even both at the same time. The digital transformation is a complex process that will fundamentally change society in the long term. It cannot be controlled but it can be essentially shaped by political measures.

One of the most fundamental risks is that legislation and political intervention addresses these changes based on what was perceived as common sense in the past. To some extent, common sense will remain unchanged, but this does not hold for all those areas where it was based on assumptions about human capabilities. If these assumptions are overturned, good practice may become bad practice and the transfer of good practice to new domains of application may lead to catastrophes. Therefore, from the perspective of legal informatics, it has become a key question whether we need a change of paradigms in law and legislation - and in case we do, how we should reflect on these changes. However far this may be from very concrete problems (like implementing patient files in compliance with GDPR), it is urgent to invest in these investigations. This paper intends to explain the impact of technology as straightforwardly as possible and thus to provide a simple starting point for a complex investigation.

\section{B. DIGITAL METATRENDS}

In all areas of life, five formative trends can be observed because of digitization:

A) Integration: The digital world becomes part of the real world $\rightarrow$ There is only ONE WORLD with physical and virtual parts.

B) Deterriorization: The place loses its importance, but it does not become completely irrelevant. $\rightarrow$ We are moving towards LANDLESS WORLD, where order is no longer determined by location.

C) Optimization (of production) according to transformation theory: Speed, precision, and complexity are ever increasing. $\rightarrow$ The loss of limitations leads to a WORLD BEYOND STRUCTURES. 
D) Optimization according to flow theory: Technology replaces organizations. $\rightarrow$ We are observing the rise of a WORLD OF PLATFORMS.

E) Optimization according to customer value theory: Radical discrimination and personalization takes place and the individual is at the same time enabled to manage herself autonomously. $\rightarrow$ The future will be the I-WORLD.

The «one world» trend arises from the digital twins of objects, companies, persons, and actions, that represent their physical twin, but may also have an existence of its own. While both twins are substantially different in nature, it makes no longer much sense to draw a clear line between them, as actions on the one may imply actions on the other. The automation of translation between both and in both directions further contributes to the increasingly inseparable integration of physical and virtual world.

The «landless world» trend means that limitations of physical distance disappear, physical proximity loses its relevance, and in many cases key activities cannot be localized anymore as they happen in a virtual environment, that is in the clouds. It is foreseeable that some companies will even try to exist without headquarters localized in a specific country. Furthermore, in a virtual setting it is hardly impossible to defend border lines and even where this must be done the strategy is necessarily different from the traditional world. The principle of the city wall must be replaced by or augmented with the principle of districts of limited complexity and the defense requires collaborations in open groups across countries, even including enemies.

The «world beyond (conventional) structures» trend means that activities take place in unparalleled speed in a highly complex setting with respect to structures, execution, and culture. Part of the control lost through complexity is regained b unparalleled precision. Thus, unstable balances of power and interests are rather typical. This leads to new governance paradigms, or rather a search for new paradigms that can control things that cannot be planned - such as, for example, high deviations which are not rare, so-called dragon kings. It remains an open question, which structures will help us in a world beyond conventional structures.

The «world of platforms» trend comprises all types of platforms from those implementing an agora, over those acting as aggregators or integrators, to those representing an alliance or a distribution channel. They include digital versions of brokers, sharing 
economy mediators, and automatic execution mechanisms like those built from distributed ledger technologies. The key issue is that organizational activity is replaced by technological executions. Consequently, in some cases the rule of law may be replaced by the rule of social or processing power. All this becomes even harder to understand as technological progress allies with storytelling and shared feeling, e.g. in the case of distributed ledger technologies shared dislike for institutions

The «I world» trend is both shaped by digitally enabled emancipation and digitally manufactured manipulation. Individuals are enabled to free themselves from traditional social structures and to submit to virtual social structures in digital social networks that are platforms for self-representation and self-localization in society, but equally serve others to gain knowledge about everyone present on the platform. While consisting of digital twins, digital social networks tend to be fake worlds and the algorithmic possibilities for profiling individuals are limited so far. Thus, the «I world» remains a realm of artificial constructs invented by computer scientists that only pretend to represent the world.

Trends 3 to 5 are developments on the shoulders of the classical production theories, that is transaction theory, flow theory, and customer value theory ${ }^{1}$. All five trends have consequences on law. The unity of physical and virtual world combines areas which are governed by different principles. We must rethink basic principles of ownership and right to use in a setting, where objects have digital twins. The most obvious challenge is created by the second trend: a deterriorized world loses the unity of order and localizability ${ }^{3}$, which leads to the question which law applies. This goes hand in hand with the trend to extend national regulation to foreign countries. The third trend creates a whole bunch of legal challenges. Structures enable the application of regulations. If structures lose their meaning, it both becomes hard to apply regulation to a unit of

1 Lauri J. Koskela, John Alfred Rooke, Sven Bertelsen, Guilherme Henrich: The TFV Theory of Production: New Developments, in Proceedings Proceedings $15^{\text {th }}$ Annual Conference of the International Group for Lean Construction, 2018 pp2-12.

2 Carl Schmitt: Der Nomos der Erde im Völkerrecht des Jus Publicum Europaeum. Duncker \& Humblot, Berlin 1950.

3 Peter Koval, Reinhard Riedl: Zur 2.0 (zweiten) Ordnung von E-Government, Proceedings IRIS 2009. 
business and the number of units increases dramatically requesting more and more resources for control. Platforms acting as market places create similar effects and challenges. Finally, the fifth trend challenges our concepts of fairness and solidarity. In an «I World» experiences are no longer shared and principles valid for all lose their legitimation.

The digital transformation changes are more than just the five trends depicted above. The digital transformation changes the relevance and the nature of production factors. Land loses some of its relevance, money undergoes unclear changes, workforce is replaced to a large extent by machines, technology becomes increasingly autonomous, and the value of education depends on the nature of the export baskets of countries (as it follows from the theory of economic complexity 4 ). This lead, among others, to a debate on whether we should introduce a third type of legal entity that would be attributed to robots. It may be fun to talk about robot rights, but the real issue is who is responsible for the actions of robots. Like for non-natural legal persons rules must be set how natural legal persons will bear responsibilities for robots in the future.

One may tell the history of economic think as a sequence of changes of focus: from land to labor \& capital to technology to knowledge to knowhow, introducing social networks as a most recent perspective. This corresponds to some extent to a change to a sequence of added perspectives in law. Currently it is rather unclear how address digital social networks in regulation, but also nondigital social networks will become a topic of debate when their relevance as production factors earns more attention.

Studying production factors from the perspective of business economics, we again meet the challenges of virtual enterprises already indicated as «world beyond structures» phenomenon. Furthermore, traditional employees are being replaced by gig workers, machines, and the crowd. Platforms may replace large sets of companies with masses of self-employed workers. This may and may not be an issue of personal choice. Therefore, regulation should set the rules for this new game. The disappearance of enterprises

4 CÉSAR A. Hidalgo, Ricardo Hausmann: The Building Blocks of Economic Complexity, PNAS 2009, 106(26). 
may concern whole sectors ${ }^{5}$, which will require a total revisiting of labor law, competition law, and other sectors. As data and reputation become dominant production factors, we are pressed to completely rethink all related laws as well. So far, we know that algorithms with high inference uncertainty are very critical and we may figure out that reputation creating evaluation may become a big problem. That alone is a lot of stuff for legal debate, but it will be the last topics to pop up here.

\section{TEN FIRING STAGES OF THE DIGITAL TRANSFORMATION ROCKET}

Looking more closely at forthcoming developments we may identify ten firing stages of the digital transformation rocket, that are fueled likewise by technological progress and cultural changes that were mostly induced either by technological change or by practices from ICT business. First and foremost, these firing stages are ignited in industry, while government continue to be busy with $20^{\text {th }}$ century digitalization. These ten firing stages may be listed as follows:

I. New resources (for labor and entrepreneurship)

II. Global distribution (of products and services)

III. Radical personalization (of products and services)

IV. Alternative valorization (of the creation of value for customers)

V. Saw tooth innovation (in industry and in people's lives)

VI. Virtual, agile, and sociocracy structures (in organizations)

VII. Ecosystem collaboration (in industry)

VIII. Networks and community business (in industry, politics, ...)

IX. Twin-based optimization \& innovation (everywhere)

X. Extreme cultures (in organizations)

5 Thomas Ramge und VikTor MaYer-Schönberger: Das Digital: Das neue Kapital - Markt, Wertschöpfung und Gerechtigkeit im Datenkapitalismus, 2018. 
Here, stages I to III correspond to the three classical production theories: stage I extends transformation theory, stage II extends flow theory, and stage III extends customer value theory. Stage IV and stage V both are relevant for economics (as they change the logic of markets) and for entrepreneurship (as they change the perspectives of business administration). Stages VI to VIII address internal and external organizational setups. Stage IX is usually affiliated with industry 4.o, but really applies to all kinds of activities, while stage $\mathrm{X}$ is far mostly a huge potential - and possibly a threat to all kinds of standards in the labor market and in society.

Ad Stage I: New resources were discussed above. They are (a) company-external workers for specific tasks, (b) crowds, and (c) intelligent machines. They imply automation, community orientation (to be able to initiate crowd activity, e.g. in the area of innovation in the form of co-creation and competitions), and augmented intelligence. Concerning (b), reputation and values tend to be key to find and employ new resources. The new resources will potentially reshape many sectors. Among others, automation has already widely transformed industry and will significantly transform mobility. And augmented intelligence will reshape many professional and academic disciplines. All three aspects of new resources may eventually totally reset the common grounds of labor and corporate law. First, it calls for a clearer definition of responsibilities, and possible for the introduction of good practice standards. Control opportunities change in several aspects which must be considered by legislation. In addition, it is likely that attribution of human properties to machines, e.g. robots, will become popular in theoretical texts and in political debates, which will create additional topics for regulation, like «machine rights».

Ad Stage II: Global distribution arises from customer-supplier matching on digital marketplaces platforms. Limitation of distribution are widely dissolved. It becomes easier to both find customers and find suppliers. Services and practices are globalized, and the context of transactions becomes multi-rational, and in legal terms multi-national. Lots of issues mentioned above will have to be discussed with respect to the regulation of such platforms. However, their market power will make it also difficult to regulate them within national context. We should rather think about new forms of transnational control of global platforms and the activities supported by them. 
Ad Stage III: Radical personalization is synonymous with radical discrimination. It means individual services and individual prices, to name the least. Customer value is maximized in both ways: customized services improve the value for customers and CRM (Customer relationship management) helps to maximize the value customers have for companies. The latter includes both tangible values directly related to cashflows and intangible values such as the reference potential of customers. All these optimizations erode shared experiences, togetherness in society, and in quite objective terms also the corner stones of traditional thinking on fairness. Among others, we will have to rethink anti-discrimination regulation. Recent research has indicated that it is highly questionable to ignore discriminatory attributes if one wants to limit statistical discrimination in decision making by machines ${ }^{6}$. In addition, personalization may even destroy whole markets, like the solidarity-based insurance markets. This calls for laws, that enable business through limiting business, which will obviously create intense political discussions.

Ad Stage IV: Alternative valorization means non-financial valorization. It can take many forms: personal data, reputation, relationships, willingness to contribute to innovation and production. Services are offered for free and valorized alternatively. In many cases this involves another group of customers who can be serviced from the non-financial values, whereby those services are then valorized financially. Potential needs for regulation so far mainly concern the use of data provided by customers. However, there is also the business model of content as value affiliated carrier of messages. When this happens, e.g. when digital content is used as a carrier for marketing messages things may become legally very tricky, even in rather conventional settings.

Ad Stage V: Saw tooth innovation is made up from two components: continuous innovation characterized by a steady stream of minor experiments and improvements plus big jump innovations resulting from technological break-through. As a result, we get something like a saw-tooth curve with respect to value generation and other measurements. This saw-tooth curve does not necessarily correspond to a long-term growth, as

6 See e.g. Muhammad Bilal Zafar, Isabel Valera, Manuel Gomez-Rodriguez, Krishna P. Gummadi: FAIRNESS CONSTRAINTS: A Flexible Approach for Fair Classification. Journal of Machine Learning, Research, No. 20, 2019. 
competition may reduce prices quickly in a market where small modifications dominate. The legal challenges arise every time when there is true technological innovation, though it takes rater long until those become business relevant. Distributed ledger technologies, i.e. blockchain+, provide an illustrative example. They combine technology innovation with value orientation and promise the replacement of institutions with technology. As such, the new technology requires a thorough legal analysis of its mechanisms plus a political debate whether institutional tasks should be outsourced to a community sharing a technology, which is in most practical application cases by its very definition anonymous.

Ad Stage VI: In the last years many so far exotic organizational structures have become popular. Most of them can be categorized as virtual, agile, or sociocracy. The dominance of hierarchy has advantages and disadvantages. The latter lead to lots of organizational innovations. For example, the disadvantages of hierarchy can be balanced with networks across the organization. ${ }^{7}$ Furthermore, agile project structures for execution have been successfully invented in software industry. They help to reduce friction losses significantly. The idea of reducing friction losses can be adapted to other organizational aspects, e.g. to feedback culture when 360 degrees feedback is minimized to very specific aspects considered to be of key importance to an organization. Such aspects are usually cultural ones and relate to values. While values tend to build up new burdens of bureaucracy, a focused, quasi-agile pursuing of these values may enable their implementation without additional frictions. Quite to the opposite, sociocracy structures are based on a broad consensus culture resulting from internal debates. Holocracy ${ }^{8}$ as a specific form of sociocracy structures replaces the traditional organizational logic of hierarchy with role structures. This in turn helps to reduce frictions and speed up change and innovation. As a specific consequence of these new forms of organization, in many organizations the responsibility for compliance may be decoupled from the

7 John P. KoTter: Accelerate: Building Strategic Agility for a Faster-Moving World, Harvard Business Review Press, 2014.

8 FREDRIC LALOUX: Reinventing Organizations: A Guide to Creating Organizations Inspired by the Next Stage of Human Consciousness, NELSON PARKER 2014. 
responsibility for organizational performance. This has consequences of legal relevance and will reshape how we handle compliance issues in organizations.

Ad Stage VII: The digital transformation relies in many settings on ecosystems around platforms ${ }^{9}$. Thereby, the creativity of participants in the ecosystem increases the value of the platform. Successful platforms are usually characterized by a dominating actor who sets clear rules, offers opportunities to introduce and valorize innovations, and are highly innovative themselves. By the very nature, this leads to the question of whether the company ruling the ecosystem may or even has to take over government roles - for example with respect to the cleaning of digital contents, i.e. the removing of criminal content. Experience shows that government can either try to negotiate directly with powerful platforms or form a cross-national coalition. However, the individual benefits of the platforms give them strong support in society, which may in turn lead to a liberalization of regulations in many sectors, for example in sectors related to tourism. As a further consequence, government roles are likely to be fundamentally questioned in society. On the contrary, as experience has shown that the owners of the core platform of an ecosystem take a major share of the economic surplus value, it is also not unlikely that political movements will request the regulation of the business models of the platforms.

Ad Stage VIII: Since reputation is a key currency in a highly transparent, digitally networked world, the successful use of communication networks becomes a key success factor. There is a common myth that communications work virally. Data about communication networks rather indicate that network structures are like Bose-Einstein molecule distributions in gases. Network science ${ }^{10}$ has thus become a key discipline in computational social sciences, that provides valuable insights how the odds for entrepreneurial success can be improved through fitting network strategies. Whether it makes sense to attribute intellectual capital to network practices is unclear. Although the networks are of key relevance for success, to the best of our knowledge this does

9 ANDREW MCAFFEE, ERIK BRYNJOLFSSON: Machine, Platform, Crowd - Harnessing our Digital Future, W.W. Norton \& Company, 2017.

10 Albert-LÁszló BARABÁSI, Márton Pósfai: Network Science, Cambridge University Press, 2016. 
not open legal questions. Nevertheless, the high economic impact of network effects leads to the educated guess that it will soon become a legal topic, too.

Ad Stage IX: As indicated above, the digital transformation leads to a universe of digital twins. They may be used for multi-aspect optimizations of the real world. Indeed, big data may even lead to the detection of optimization aspects without a natural realworld perspective or explanation. Furthermore, creative design may directly deal with digital twins to prepare innovations. Recently, within big data research two new research topics have emerged that relate to the «emancipation» of digital twins from real world experience. One research topic is research on how to explain the results of big data, the other research topic is inference uncertainty. Both are of high relevance. A third research topic results from the wish to understand the nature of algorithms without mathematical expertise. It deals with the question of how to classify algorithms in a socially relevant, but equally mathematically precise way? They key problem there is that mathematics has so far not invented proper measures. It is unclear whether it ever will. However, it is possible to characterize features of the use of algorithms that may be socially harmful. ${ }^{11}$ Nevertheless, the benefits of optimization and innovation based on digital twins are manifold and lead both to economic growth and social innovations. We need to do research on legal reasoning within the space of digital twins. As a straightforward example, big data methods to identify causal relationships will have to be taken up in court practice.

Ad Stage X: So far, there are coffin corners in business economics, in which companies do not survive for long. They are characterized by extreme orientations. For example, extreme innovation or, to the contrary, extreme optimization of resources do not allow mid-term survival12. The same holds for extreme orientation towards competition, or to the contrary, for extreme orientation towards social togetherness. Digital tools help us counterbalance extreme orientations. For example, networks across a company ori-

11 Cathy O’NeiL: Weapons of Math Destruction: How Big Data Increases Inequality and Threatens Democracy, Crown, 2016.

12 DAvid HurST: The New Ecology of Leadership: Business Mastery in a Chaotic World, Columbia Business School Publishing, 2012. 
ented towards resource optimization may help to keep innovation alive in this company. For example, balanced scorecards may help to keep resource under control in a company oriented towards innovation. Or, as another type of example, the lack of social togetherness may be compensated by an orientation towards a specific shared value ${ }^{13}$. Since coffin corners relate to optimizations, they may become attractive positions if they turn out to be no longer deadly. Extreme organizations may even widely displace companies with a balanced setting/position. This will open lots of legal questions how to regulate extreme business.

Ad other/further Stages: The ten firing stages of digital transformation as they were depicted above, do not cover the whole area of digital transformation. There are lots of further types of innovation. We already foresee follow-up transformations. When socalled «mobility 4.0» turns out to be one where individual driving is banned from cities and motorways, then cars may turn into entertainment places as well as stations in the customer journeys. This will not only effect car industry, data business, and entertainment industry, but may call for new regulations of mobility within cities, e.g. when travelling in a car becomes a side-effect of other services and thus increases traffic beyond what can be handled, even with the use of artificial intelligence for the steering of traffic. Thus, right now there is neither an endpoint of nor a clear borderline for digital transformation. We are participating in a change process that questions the foundations of our society. We rather should start now to analyze this change and think about legal paradigm changes.

\section{TRENDS IN GOVERNMENT}

The gap is widening between those digital optimizations of execution that are possible and those digital optimizations that have been implemented. Our understanding of

13 SAlim Ismail, Yuri VAn GeEst, Michael S. MalonE: Exponential Organizations: New Organizations Are Ten Times Better, Faster, and Cheaper Than Yours (and What to Do About It), Audible Studios, 2015. 
what should be done in e-government has increased. In addition, technological progress has created new perspectives for e-government. Both contribute to the widening of the gap as the implementation progress is much slower.

There is a long list of innovations topics that have been hardly implemented if at all: personalized portals with $" \mathrm{C}_{3} \mathrm{RM}$ : citizen-controlled citizen relationship management», tailor-made, situated information providing for citizens, participatory policy making with open innovation and co-creation as well as with authentic simulations ${ }^{14}$, infrastructure for the digital transformation of industry and society like trust \& confidence services, intelligent control of mobility services and built infrastructure (e.g. to control the microclimate in cities and the use of natural resources), or open collaboration in operational tasks in the sense of «open data - open processes - open services».

Viewing the domain of e-government from an engineering and design perspective, it is about digitization of resources, integration of resources, and sharing of resources., because this creates new degrees of freedom for the organization of public administration. There are more than a dozen technological solution patterns that are the corner stones of e-government that have only in parts been implemented. These patterns include information portals, transaction portals, electronic identity, integrated transaction processing, integrated registers, communication buses, process automation, resource brokers, shared service centers, open government data, etc. They all represent a different form of integration and sharing of digital resources. The main hindrance blocking their implementation is that it is hard to communicate that (a) the integration and sharing of digitalized resources creates new freedom for organizing institutions and that (b) freedom is often perceived as a threat.

The key legal challenge is that inefficiency positively correlates with corruption. Thereby, it is relative inefficiency and not absolute inefficiency that counts, plus possibly the speed of change and transformation. The slow implementation of the digital transformation in government thus creates new corruption risks in many countries. 
New approaches to governance and control are needed to postpone the emergence of corruption.

One possibility to think further in e-government is to move from the integration and sharing to the creation of platforms, or to really rebuild parts of government as a platform ${ }^{15}$ for the solution of problems of society. This would involve industry, civil society, and citizens and support their effective collaboration. And it might thus free new resources for implementation. However, in most countries such a platform would require a constitutional or/and legal foundation.

City government is a specific case of government with challenges. The topics of good governance have been the same for centuries: They comprise jurisdiction, city administration, the interplay with the hinterland and city, the allies with other cities, and the city architecture. However, the tasks for creating a good city architecture have changed. While in the past city governors were concerned about the interplay between built space and social space, today they must care about the interplay in the triangle between built space, social space, and digital information space. This calls for new strategies, and possibly as well for legal interventions.

Of course, lots of the trends in industry and society create challenges in government. They, too, ask for creative approaches, both in execution and in its legal foundations. The ever-increasing complexity of government makes it clear, that slow progress in digital transformation is no long-term option.

\section{E. THE DANGERS OF DIGITAL TRANSFORMATION}

The digital transformation brings with its new dangers, risks and special challenges, as any technologically initiated progress did before. These dangers include

1. Cyber risks including cybercrime and cyberwar

2. Limited trustworthiness and reliability of digital instruments

15 Tim O’REILlY: WTF? What's the Future and Why It's Up to Us?, Random House Business, 2018. 
3. Discrimination of those who lack the capability to learn new digital skills

4. Adverse side-effects of the positive effects

The digital transformation is also an enabler for semi-silky actors, criminals, and people with fundamentalist attitudes. They misuse data, they cause malfunctions and they can paralyze our society. A successful fight against cyber-crime and emerging forms of nearcyberwar requires permanent development of defense tools, some transparency about attacks, a semi-open collaboration that includes the collaboration with other countries (and even enemies), shared legal standards, activities to collect information in darknet, highly skilled defense teams, and a proper governance and control of defense activities. We need legal rules for IT forensics, the training of defense teams, the admissible activities in darknet, the identification of aggressors, and for our dealing with identified vulnerabilities. And we need legal rules how to control those who are here to defend our security.

But there are also dangers without attacks. Digital instruments can fail. They can be used in an unskilled, improper way. And their use may have far reaching consequences that are hard to forecast. This calls for clear rules for the design and the use of advanced digital tools in critical settings.

Using digital tools properly can be highly demanding. It requires both some commitment to start using them and rather advanced digital skills. In addition, it may lead to an extended surveillance. This may reduce the job opportunities of people with a lack of cognitive skills or with mental diseases. Again, it will be necessary to define rules on which mastership of digital tools can be expected from employees and from citizens in general.

The topic of adverse side-effects of seemingly positive ones is a huge one. For example, digital tools may create transparency that we are not prepared for. This transparency may help to avoid mistakes, it may also undermine the individual commitment to responsible conduct. Further, transparency may erode solidarity and lead to the necessity for new regulations. Most dangerously, it may create the illusion that we know something where we only are able to observe certain aspects. More generally, the capabilities created through digital tools enable lots of things that are very promising and positive 
for the common good, but they equally enable lots of things that are potentially devastating for our society.

\section{F. THE TRUE CHALLENGES}

It follows from above that critical aspects of life, like the labor market, are about to change significantly. In many areas cultural values will erode, change, or newly emerge, which will require legal changes. Social practices and political attitudes will change, attitudes towards institutions / institutional processes as well as towards individual rights. New institutional settings will require legislative foundations. New work practices will require the formulation of clear principles of what is admissible and what is not. Questions of governance and responsibility will need much more attention than in the past. In some areas multi-national solutions are a must. In other areas we shall need new paradigms to deal with multi-rational settings. These observations call for a rather fundamental legal analysis of the ongoing digital transformation. We have enough time to develop proper principles and paradigms if we start now, but we have not enough time to ignore fundamental changes until their manifestation can be empirically and broadly observed. That is, one key challenge is to come up with a theoretical background for future regulation without much empirical evidence. This is particularly challenging whenever strong beliefs contradict optional future developments.

However, this is only part of the story. Another key challenge - and possibly the ahead - is the growing popularity of breaking rules to move faster ${ }^{16}$. Parallel to this trend one may observe a loss of common grounds in many sectors because the world is becoming increasingly complex. Common sense loses most of its relevance as the digital tooling creates conflicts between the transversal logic of digital transformation and the traditional logic of domains. This does not hold factually but it holds in many societal contexts. Consequently, breaking the rules does not get punished where it should, because it is hardly detected in settings where order structures and their logic reasoning are upside-down. In a way, regulation becomes the only protection of society, while the

16 Jonathan Taplin: Move Fast and Break Things: How Facebook, Google, and Amazon Cornered Culture and Undermined Democracy, Little, Brown, and Company, 2017. 
execution of rules either becomes less and less successful (due to increased complexity and a slow progress in the digital transformation of government) or is delegated to machines, platforms, or service providers far abroad. It becomes evident that it is the interplay of regulation, government, and common grounds in society that is needed for good governance. So far it is fully unclear how we compensate for the loss of common grounds in society.

But this, too, does not complete the story. We are observing a fundamental conflict between the Silicon Valley mindset, which is founded by the belief that stepwise technological fixes can solve all problems of the world, and our common experience that neither organizations nor societies follow the rules of mechanics, but rather react in highly surprising ways to interventions. Some problems can be treated and solved as niche problems, others simply can't. They require a holistic approach and a deep understanding of the dependencies and interactions. Looking at the lots of new startups in the cybersecurity market reveals a fundamental weakness: They are focused on niche problems and sell their services as they would provide holistic solutions, because both makes economic sense. However, it is usually very hard to decide which combination of cybersecurity services will provide considerable security to an enterprise, if this is possible at all. Unfortunately, the required holistic solutions neither comply with the Silicon Valley mindset, nor with the economic necessities of starting a new business. We must therefore decide in many areas, which degree of holistic good practice shall mandatory in the future. This is necessary in cybersecurity, but not only there.

Finally, we should avoid adopting the Silicon Valley mindset in legislative activities. In the future, we shall need more than specific patches for laws that no longer meet the requirements of a digital economy and society. For some of the challenges listed in this article we shall need paradigmatically new approaches to regulation. 